Review Article

\title{
A Systematic Review of the Anti-Inflammatory and Immunomodulatory Properties of 16 Essential Oils of Herbs
}

\author{
Xu Zuo $\mathbb{D}^{\text {, }}$, Yinuo Gu $\mathbb{D}^{\mathrm{D}}$, Chao Wang, Jinrong Zhang, Jing Zhang, Guoqiang Wang, \\ and Fang Wang \\ Department of Pathogeny Biology, College of Basic Medical Sciences, Jilin University, Changchun 130021, China \\ Correspondence should be addressed to Fang Wang; wf@jlu.edu.cn
}

Received 10 September 2020; Revised 26 November 2020; Accepted 28 November 2020; Published 7 December 2020

Academic Editor: Roberto K. N. Cuman

Copyright (c) $2020 \mathrm{Xu}$ Zuo et al. This is an open access article distributed under the Creative Commons Attribution License, which permits unrestricted use, distribution, and reproduction in any medium, provided the original work is properly cited.

\begin{abstract}
Background. Inflammation is a host defense mechanism in the body after it is infected and damaged. If inflammation is not treated in time, then it may cause a variety of diseases, such as cancer and autoimmune diseases. Herbal essential oils are natural extracts that can suppress inflammation effectively and are expected to be used in therapeutic drugs for anti-inflammatory diseases in the future. Aim of the review. We review the anti-inflammatory and immunomodulatory effects of essential oils derived from 16 herbs. Materials and methods. We searched the literature of the fields of anti-inflammatory and immunomodulatory herbal essential oil activity published in English within the past five years via databases (PubMed, EMBASE, Scopus, and The Web of Science). Results. A total of 1932 papers were found by searching, and 132 papers were screened after removing duplicates and reading article titles. Fifteen articles met the requirements to be included in this review. Among those selected, 11 articles reported in vivo research results, and 10 articles showed research results. Conclusion. Essential oils extracted from herbs can reduce inflammation by regulating the release of inflammatory cytokines involved in multiple signalling pathways. Herbal essential oils are expected to be developed as anti-inflammatory drugs.
\end{abstract}

\section{Introduction}

Inflammation is a defense mechanism against infection and tissue damage [1]. Inflammation and proinflammatory cytokines, reactive oxygen species (ROS), lipid extraction medium: arachidonic acid (AA), hydrolases, transcription factors, etc., are closely related [2]. When not stopped in time, inflammation can cause diabetes and cancer [3], arthritis, Alzheimer's disease, atherosclerosis, cardiovascular disease, eye diseases, and autoimmune diseases, including inflammatory bowel disease [4]. Some clinical and physiopathological data also showed that inflammation could also affect children with inflammatory bowel disease, uveitis, and juvenile idiopathic arthritis by slowing their growth in height and weight [5]. Inflammation seriously affects people's lives.

Inflammation can usually be classified into two categories according to its course: acute inflammation and chronic inflammation. The immune system plays a key role in determining whether inflammation is acute or chronic.
The activation of inflammation is closely related to immune cells and biological molecules. In particular, innate lymphoid cells (LCs), with multiple immune functions, play important roles in inflammatory diseases [6]. Infiltration of macrophages and neutrophils is a feature of acute inflammation, and infiltration of T lymphocytes and plasma cells is a feature of chronic inflammation [7]. These cells play important roles in the inflammatory response.

Steroidal and nonsteroidal anti-inflammatory agents are commonly used in the clinical treatment of inflammatory diseases. Long-term use of these drugs can cause serious adverse reactions, such as gastrointestinal tract, cardiovascular, and liver abnormalities [8]. Finding a safe and effective drug to control inflammation has always been a challenge. In recent years, Chinese herbal oils have been rated as the safest, most promising anti-inflammatory drug candidate [2].

Recently, it has been shown that a variety of active components of traditional Chinese medicine regulate the 
body's immune state to attenuate inflammation [9-11]. Volatile Chinese medicine oils are mixtures of multiple compounds [12]. The main components are monoterpene and sesquiterpene, among others, with anti-inflammatory, antibacterial, acaricidal, antiviral, sedative, antianxiety, and antidepression properties [13]. The anti-inflammatory effect of volatile oils of traditional Chinese medicine is mainly realized through the regulation of cyclooxygenase (COX) and induction of nitric oxide synthase (iNOS) and a variety of cytokines, which play roles in the inflammation process [14]. In recent years, essential oils of traditional Chinese medicine have been described as potential sources for the development of new drugs.

\section{Materials and Methods}

The review was conducted following the Preferred Reporting Items for Systematic Reviews and Meta-Analyses (PRISMA) statement [15].

2.1. Search Strategy. Every publication in English that was reviewed for this study was extracted from the PubMed, Scopus, EMBASE, and Web of Science databases restricted to the Medical Subjects Headings Index (MeSH/DeCS) to April 2020. The search was based on different combinations of the following keywords: "essential oil", "oils, essential," "essential oils," "volatile oils," “inflammation," "inflammations," "innate inflammatory response," "inflammatory response innate," and "innate inflammatory responses." Furthermore, we reviewed the references in the selected articles for additional reports not included in the original article search.

2.2. Study Selection. The two authors independently extracted and proofread the titles and abstracts of each article. The inclusion criteria were the effects of volatile oils and the active components of traditional Chinese medicine on inflammatory diseases, including in vivo and in vitro models and the possible mechanisms of action. The authors excluded articles based on the following criteria: review articles, meta-analyses, abstracts, editorials/letters, conference proceedings, case reports, studies in humans, and articles published more than 5 years ago. The differences of opinion between the two authors were independently decided by the third author.

2.3. Data Extraction. One author summarized the data from the article, and the other author examined it. Table 1 summarizes the following information from the in vitro experiments: the source of the essential oil, species, inflammation type, test index, cell line, the proposed mechanism (s) of biochemical effects, and conclusion. Table 2 summarizes the following information from the in vivo experiments: the source of the essential oil, species, inflammation type, test index, experimental animal species, the proposed mechanism (s) of biochemical results, and conclusion.
2.4. Methodological Quality Assessment. Preclinical in vivo studies of bias risk and quality were based on an optimized checklist [15, 31]. This survey mainly involved the randomization of animal feeding and distribution, blind administration, blind results, and other factors used to evaluate the included methodology.

2.5. Data Analysis. Due to the heterogeneity of this study, pooled statistics and meta-analysis were not used. The data analysis is presented in narrative form.

\section{Results and Discussion}

3.1. Search Results. Figure 1 presents a flow chart of the search; 1154 articles were identified (PubMed: 115, EMBASE: 567, Scopus: 518, and Web of Science: 732) after duplicate articles had been deleted. After reading the title, we removed articles that were unrelated to essential oils and inflammation and 132 were thus retained. Finally, 15 articles were selected after full-text browsing, among which 12 articles included in vivo experiments, 9 articles included in vitro experiments, and 7 articles included in vivo and in vitro experiments.

3.2. Study Characteristics and Description. There were 12 in vivo and 9 in vitro experiments. The influence of essential oil and its components on the inflammatory disease model was investigated in vitro using an inflammatory disease model constructed with a variety of cell lines. Using in vivo experiments, 12 articles studied the effects of traditional Chinese medicine essential oils and their active components on a variety of inflammatory disease models based on mice, rats, or rabbits. The oils showed a significant inhibitory effect on inflammation. Part of the chemical structure of traditional Chinese medicine essential oils is shown in Figure 2. Of the studies examined, 9 articles were from China, 2 were from India, 1 was from Iran, and 1 was from South Korea.

In vitro experiments were performed in human aortic endothelial cells (HAECs), a human keratinocyte cell line (HaCaT), the THP-1 cell line, mouse primary splenocytes and peritoneal macrophages, RAW264.7 cells, and ANA-1 cells. Many in vitro experimental methods were reported in the articles, including immunohistochemical analysis, apoptosis assay, and histological assessment, and proinflammatory cytokines were quantitatively measured by ELISAs, with western blotting and RT-PCR used to detect the expression of various proteins and mRNA, respectively.

In vivo models of inflammation included dextran sulfate sodium (DSS) induced intestinal inflammation, TPA-induced mouse skin inflammation, carrageenan-induced paw oedema, xylene-induced ear oedema model, 2, 4-dinitrofluorobenzene (DNFB)-induced allergic contact dermatitis (ACD), and ischaemic renal rat models. A number of experimental methods were used in the in vivo experiments, including the acetic acid-induced writhing test, oxytocininduced dysmenorrhea in mice, formalin test, complete Freund's adjuvant-induced overt pain test, carrageenaninduced mechanical hyperalgesia test, evaluation of cytokine 


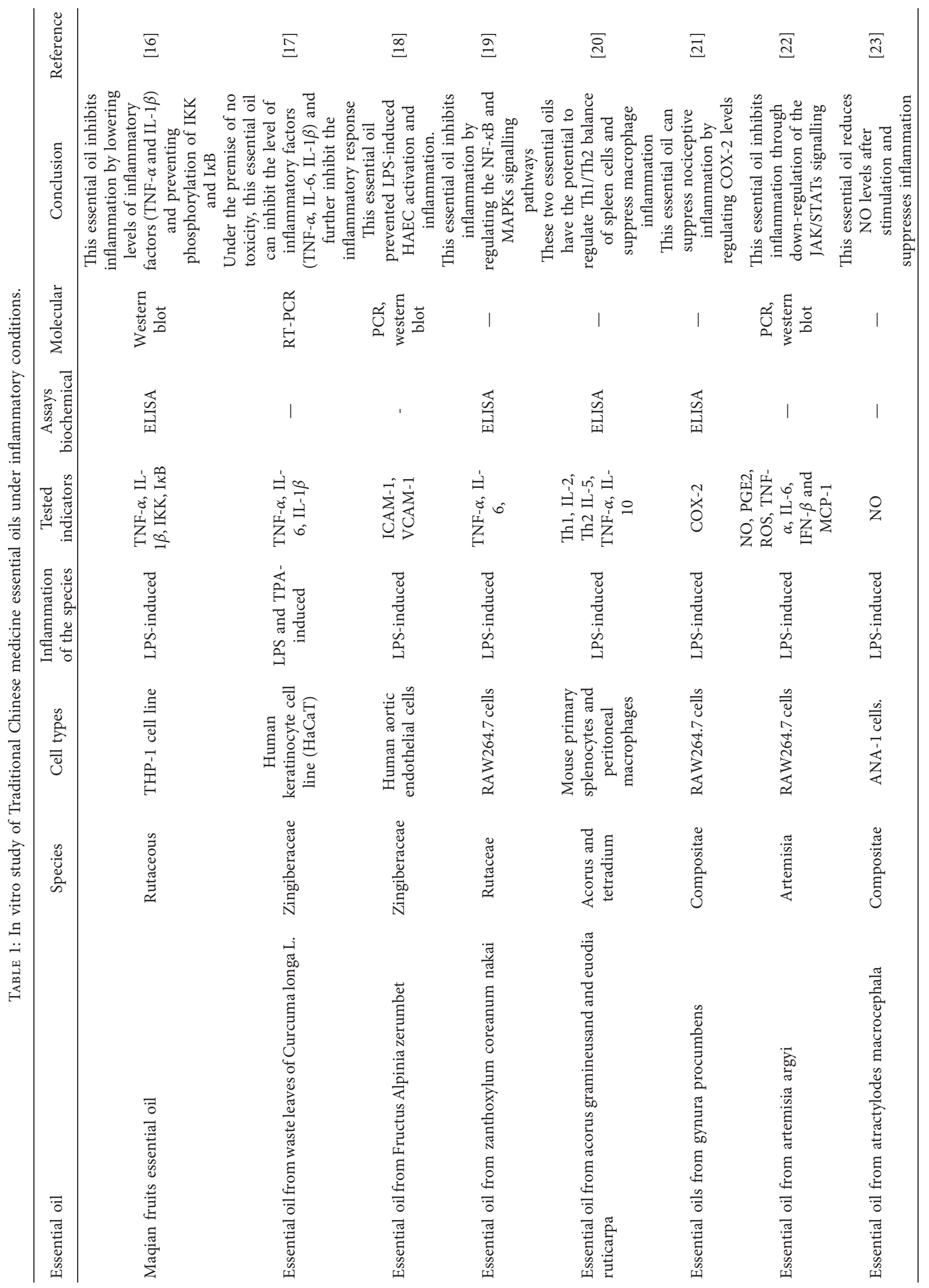




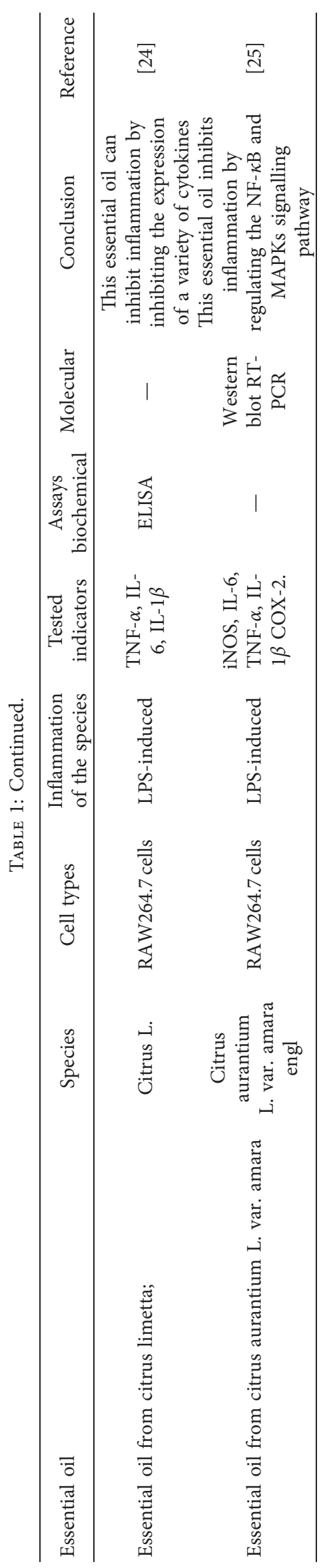




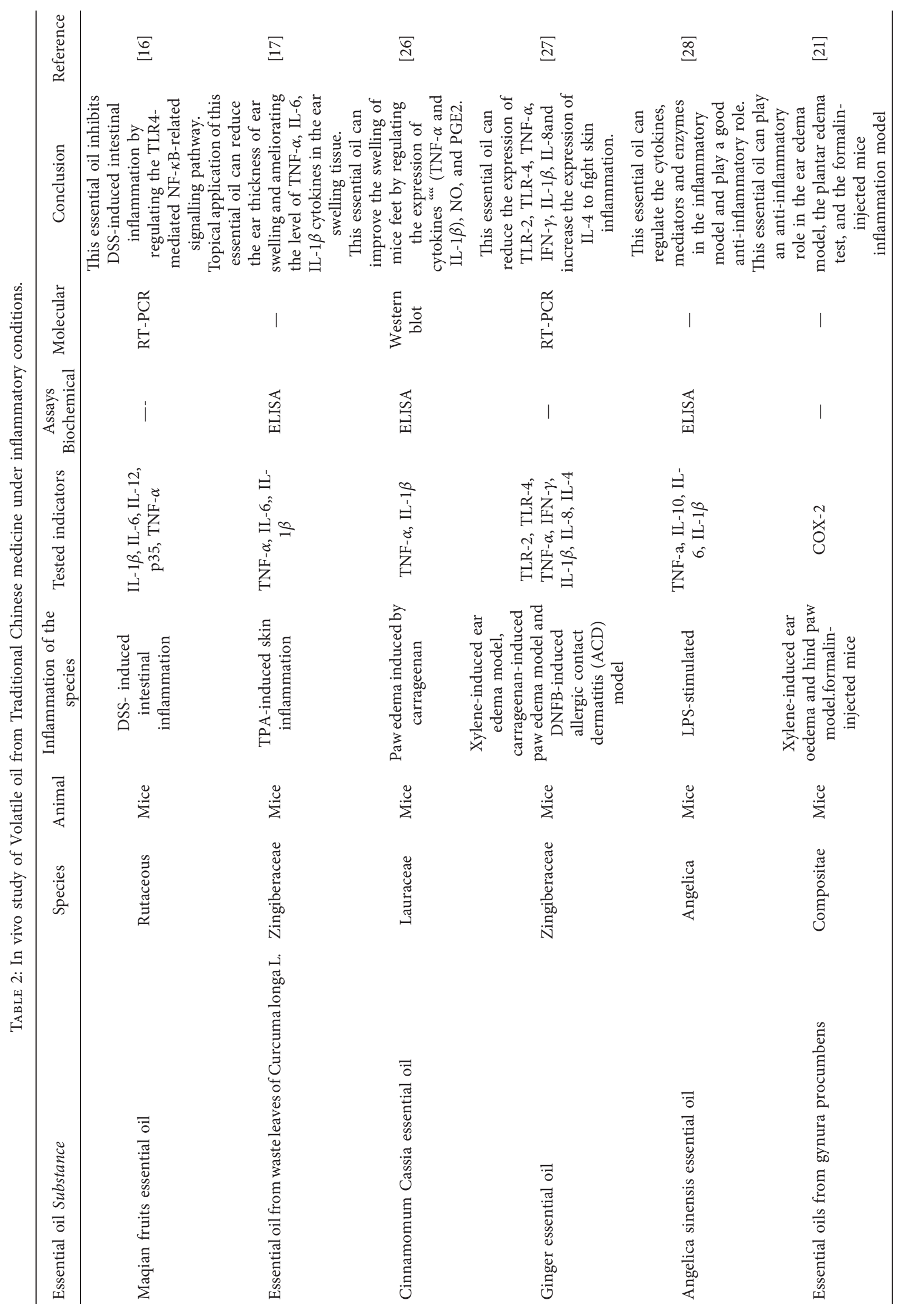




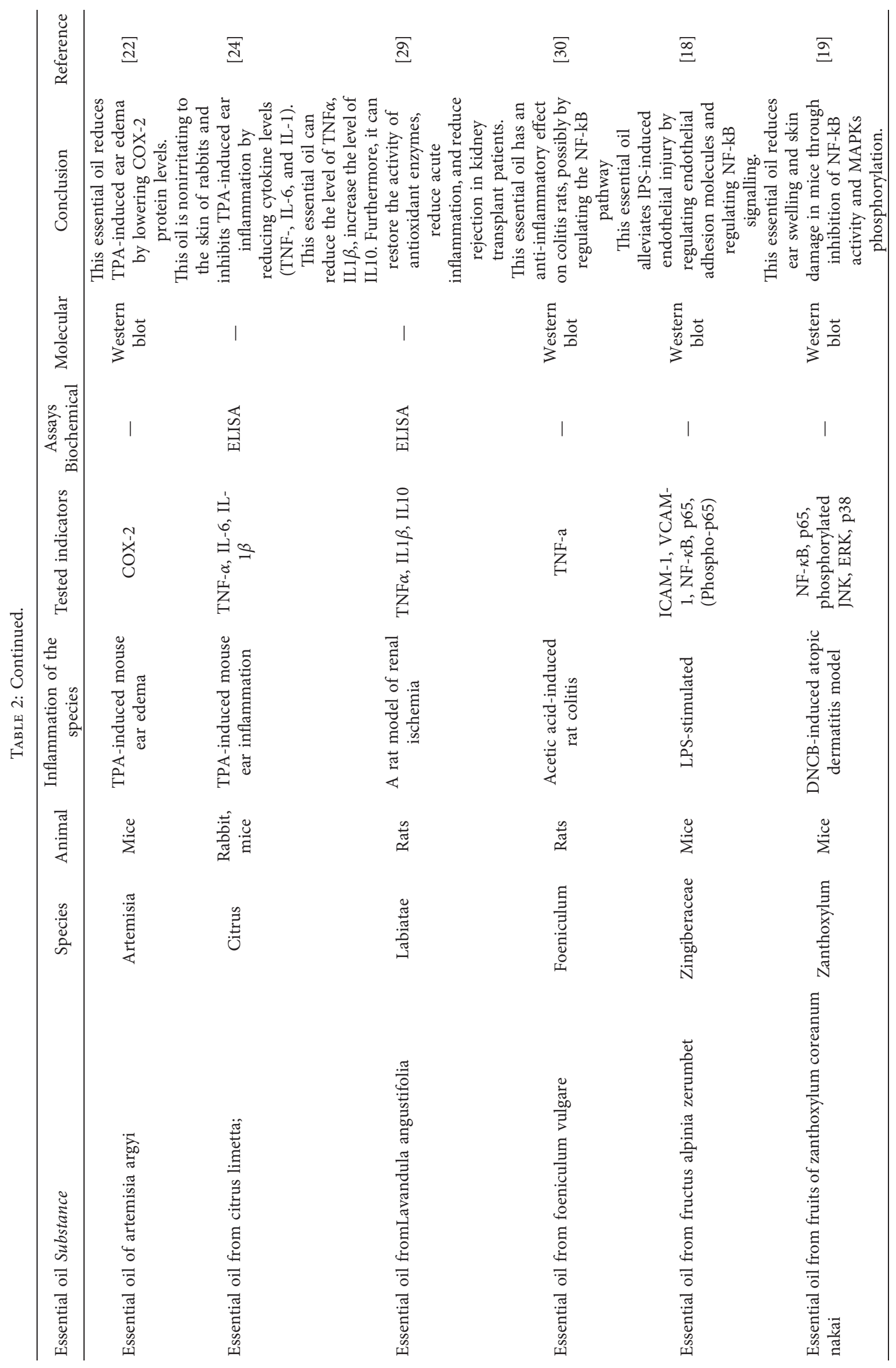



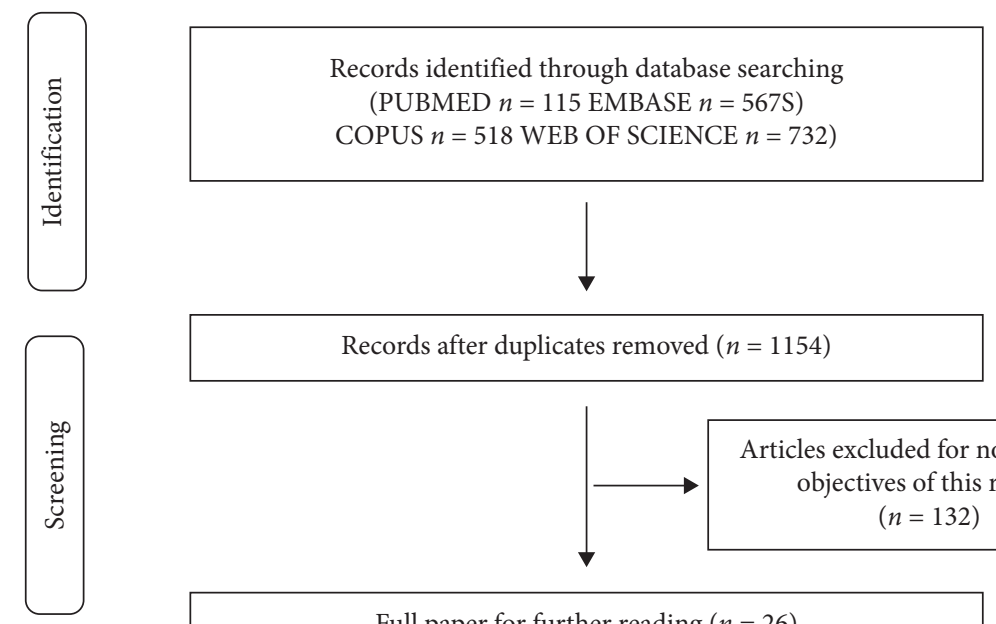

Records after duplicates removed $(n=1154)$
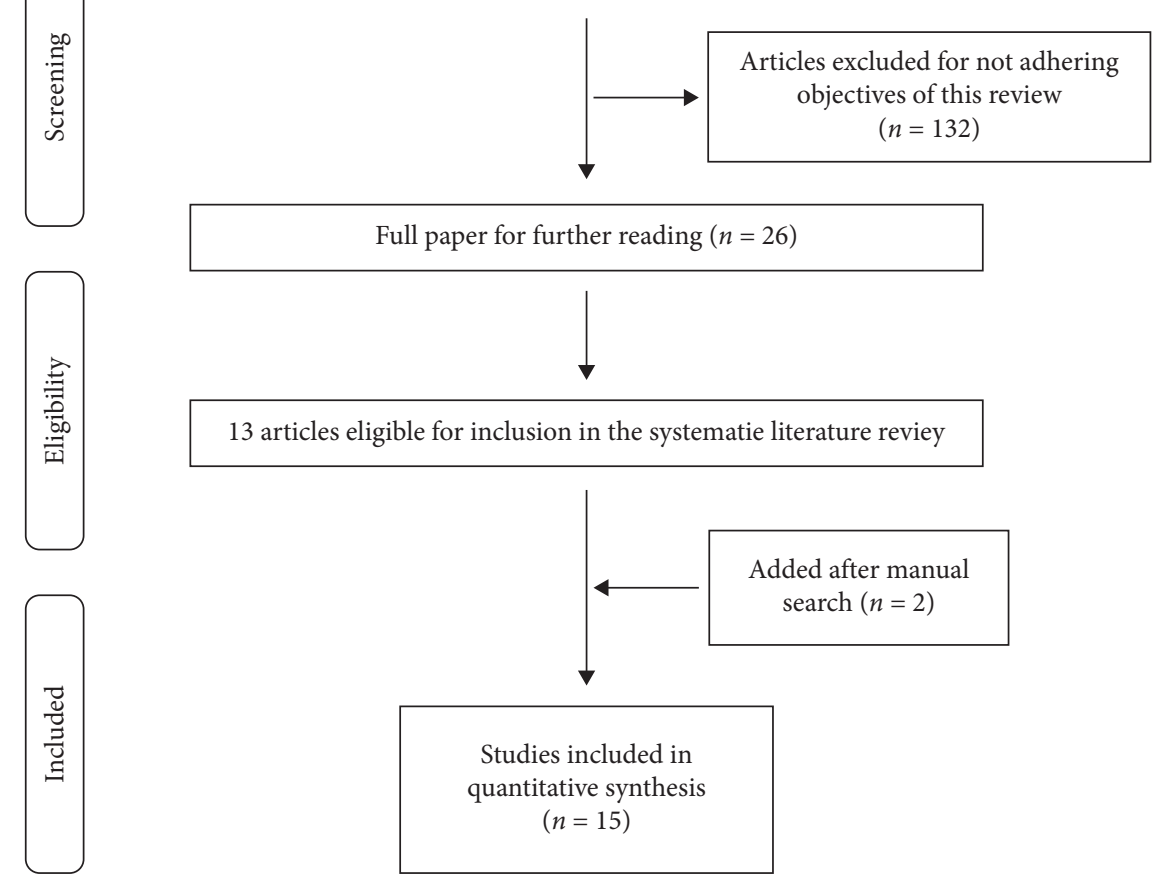

FIgURE 1: A flowchart of the literature search and selection in this review is described in detail.<smiles>CC=C1CC=C(C)CC1</smiles>

Terpinolene<smiles>CC1=CCC(C(C)(C)O)CC1</smiles>

$\alpha$-terpineol<smiles>C=C(C)[C@H]1CC=C(C)CC1</smiles>

Limonene<smiles>O=C/C=C/c1ccccc1</smiles>

(E)-Chinnamaldehyde<smiles>C=C1CCC[C@]2(C)Cc3occ(C)c3CC12</smiles>

Atractylone<smiles>CC1=CCC2CC1C2(C)C</smiles>

(-)- $\alpha$-Pinene<smiles>CCC/C=C1\OC(=O)c2ccccc21</smiles>

Ligustilide

FIGURE 2: Chemical structure of volatile oil in Chinese traditional medicine.

levels by ELISAs, and western blot analysis. Through the aforementioned experimental methods, it was further shown that Chinese traditional medicine essential oils have obvious attenuating effects on various kinds of inflammation.
3.2.1. Anti-Inflammatory Effects of Traditional Chinese Medicine Essential Oils In Vitro. An inflammatory response is a symptom of many diseases caused by bacteria or viral infection, physical stimuli, chemical stimuli, and trauma and 
is a complex biological response to harmful stimuli [32]. Lipopolysaccharide (LPS) is a component of the cell wall of Gram-negative bacteria that causes inflammation by activating MAPK, nuclear factor-kB (NF-kB), and activator protein-1 (AP-1) signalling pathways [18]. In the inflammatory response of cells, COX-2 inhibition exerts a strong anti-inflammatory effect [33]. COX-2 overexpression can also be used as an inflammatory model.

The essential oil from Gynura procumbens (GPEO) and its three active components, $a$-pinene [34], 3-carene, and limonene, [35] can inhibit the inflammatory cell infiltration induced caused by noxious stimuli [36]. They have a significant pharmacological effect on the migration of RAW264.7 macrophages induced by LPS and inhibit COX-2 overexpression [37]. Because of its good permeability and anti-inflammatory properties, GPEO is a great treatment for relieving skin redness or treating poisonous insect bites.

The essential oil from Artemisia argyi (AAEO) [38] can inhibit the release of inflammatory mediators (NO and PGE2) and the expression of cytokines (IL-6, IFN- $\beta$, IL-10, and MCP1). AAEO inhibited the expression of iNOS and COX-2 at the transcription level without affecting its activity [39]. Moreover, AAEO also inhibited the phosphorylation of STAT1 (Tyr701) and STAT3 (Tyr705) and downregulated JAK/STAT signalling and ROS scavenging. ROS production plays a key role in the activation of JAK/STATs in macrophages. According to a previous report, essential oils scavenging ROS have antioxidant activity; therefore, the inhibitory effect of AAEO on JAK/ STAT may be due to its antioxidant activity, especially its effect on ROS production $[14,40]$.

In intestinal inflammation diseases, the levels TNF- $\alpha$, IL-1 $\beta$, IL-6, and IL-12 are significantly higher than those in noninflamed intestines [41]. Intestinal inflammation is often accompanied by mucosal immune system disorders. Researchers found that Maqian fruit essential oil (MQEO) reduced LPS-stimulated expression of TLR4 in THP-1 cells and effectively suppressed the production of IL- $1 \beta$ and TNF- $\alpha$ in a dose-dependent manner, and in the past, researchers found that curcumin and ellagic acid [42], which are naturally occurring plant phenols, exhibit anti-inflammatory activity by preventing $\mathrm{I} \kappa \mathrm{B}$ degradation [42]. Linalool is the main component of the essential oil from the blossoms of Citrus aurantium L. var. amara Engl (CAVAO) [43]. It can suppress inflammatory symptoms by suppressing $\mathrm{NF}-\kappa \mathrm{B}$ P65 translocation. It blocks the phosphorylation of IKK and $\mathrm{I} \kappa \mathrm{B}[44,45]$. The anti-inflammatory effect of MQEO may also be related to the TLR4-mediated NF- $\kappa \mathrm{B}$ signalling pathway. These two kinds of traditional Chinese medicine essential oils have very good prospects in intestinal inflammatory diseases.

Essential oil extracted from fructus Alpinia zerumbet (EOFAZ) [46] changes the expression of ICAM-1 and VCAM-1 and promotes the adhesion of inflammatory cells. Target genes [47] for NF- $\kappa$ B include ICAM-1 and VCAM-1. Atherosclerosis is characterized by chronic systemic inflammation and the formation of large and medium-sized atherosclerotic plaques. The amelioration of inflammation plays an important role in the treatment of this disease $[48,49]$. Endothelial cells can promote the adhesion of inflammatory cells by changing the expression of ICAM-1 and VCAM-1 [50], which is believed to play an important role in the occurrence of inflammation and the pathogenesis of atherosclerosis [51]. EOFAZ may serve as a new drug source for the treatment of atherosclerosis.

Mast cells play important roles in allergic inflammation [52]. The essential oil from Zanthoxylum coreanum Nakai (ZCO) has been shown to effectively inhibit mast cell degranulation and reduce the level of IL-4, a key inflammatory factor in allergy symptoms [53]. Thirtyseven active ingredients identified from ZCO, mainly $\beta$ ocimene and (-)- $\alpha$-pinene, can alleviate allergic inflammation by suppressing the MAPK and NF- $\kappa \mathrm{B}$ signalling pathways. ZCO has good transdermal absorbability [54] and will likely be used to treat allergic inflammation in the future.

The skin is the largest sense organ in humans and plays an important role in immune defense [55]. An inadequate immune defense can cause some types of dermatitis. Skin inflammation is closely related to the levels of cytokines and reactive oxygen species [56]. The essential oil from Curcuma longa (EOCl) [57] significantly reduced the levels of TNF- $\alpha$, IL-6, and IL- 1 in inflammatory models. The main components of EOCl are terpinolene and phellandrene. The essential oil from Citrus limetta Risso peels (Clp-EO) is also a candidate for treating skin inflammation [58]. Its main ingredient is limonene, which is not cytotoxic. It can inhibit the levels of TNF- $\alpha$, IL- 6 , and IL- $1 \beta$ in inflammation models and can reduce the levels of ROS under oxidative stress conditions. These two kinds of Chinese herbal essential oils can reduce the levels of various oxidative stress markers. They play antioxidant roles, including a therapeutic role in skin inflammation.

The essential oils extracted from crude A. macrocephala (CA) and bran-processed A. macrocephala (BA) are CAEOs and BAEOs [59]. The main components of these two essential oils are Atractylone [60]. Atractylone effectively inhibited the level of NO in an allergic rhinitis (AR) model [61]. Atractylone can reduce AR biomarkers and exert a certain anti-inflammatory effect. CAEOs and BAEOs not only exert anti-inflammatory effects but also inhibit gastric cancer, intestinal cancer, and liver cancer cells [62]. These two essential oils have the potential to treat AR. Essential oils can be inhaled into the nasal cavity, which is the best route of administration [63]. They will be the preferred raw materials for the treatment of AR in the future.

Acorus gramineus (AG) and Evodia ruticarpa (ER) steam-distilled essential oils (SDEOs) are rich in phytochemicals such as total flavonoids, polyphenols, and saponins. The balance between T helper 1 (Th1) lymphocytes and T helper 2 (Th2) lymphocytes is important for human health [64]. These two essential oils can regulate the Th1 and Th2 immune balance and inhibit the TNF- $\alpha /$ IL-10 cytokine ratio during the inflammatory response of macrophages, showing a certain anti-inflammatory ability [65]. 
3.2.2. Anti-Inflammatory Effects of Traditional Chinese Medicine Essential Oils In Vivo. For in vivo tests of essential oils of traditional Chinese medicine with respect to anti-inflammatory diseases, most articles reported the use of chemical reagents to induce inflammation. Essential oils of Chinese medicine have inhibitory effects on inflammation induced by chemical agents. They play inhibitory roles in inflammatory diseases such as skin inflammation and intestinal inflammation, 12-O-tetradecanoylphorbol-13-acetate (TPA) [66] can be used to induce skin inflammation in vivo [67]. After 4 hours of skin exposure to TPA, COX-2 expression in the skin increased significantly, and ear thickness increased significantly. Histological analysis of mouse ear slices can be used to observe the occurrence of inflammation. Skin oedema after successful modelling is obvious, showing many infiltrating neutrophils. This ear oedema model is an ideal in vivo model for screening anti-inflammatory drugs. The essential oils from Gynura procumbens, Citrus limetta Risso, Curcuma longa, and Artemisia argyi use the TPA-induced inflammation model in mice, and all four essential oils can reduce the degree of ear swelling in mice [21]. The overproduction of oxidative markers can be regulated by reducing the expression level of COX-2 protein-improved levels of proinflammatory cytokines (TNF- $\alpha$, IL-6, IL-1). Immunohistochemical methods have shown that Chinese medicine essential oil can restore histopathological damage to a great extent. It plays a significant role in inhibiting inflammation.

In the essential oil extracted from Cinnamomum cassia Presl, a variety of substances, such as benzenepropanal, (E)cinnamaldehyde and turmerone, were detected [26]. They can reduce the inflammatory response after different kinds of stimuli. After formalin and CFA were injected into the foot of the mice [68], the mice that took oral oil significantly improved their paw licking behavior. At the same time, the levels of TNF- $\alpha$, NO, IL-1 $\beta$, and PGE 2 in paw oedema tissues induced by carrageenan were reduced in a dosedependent manner [68]. EO showed an inhibitory effect on acetic acid-induced abdominal contraction. The expression of COX-2 and iNOS in the skin tissues of the feet of mice with essential oils decreased. This shows that this essential oil has a certain anti-inflammatory effect.

Ischemia reperfusion (I/R) injury is a major problem that occurs after a kidney transplant, and it can lead to inflammation [69]. Lavender oil has a certain anti-inflammatory effect on a rat kidney I/R injury model [29]. Oral essential oil can improve renal function by reducing the level of oxidative stress in the pathological cascade caused by renal $\mathrm{I} / \mathrm{R}$. Lavender oil can effectively resist renal I/R injury by targeting oxidative stress and apoptosis.

In in vivo models of intestinal inflammation, DSS is generally used to stimulate the intestinal epithelial cells of mice [70], causing mice to produce various symptoms of intestinal inflammation, such as diarrhoea and granulocyte infiltration. Maqian essential oil can reduce MPO and MMP-9 levels in inflammatory tissues, and its anti-inflammatory activity may be related to the NF- $\kappa \mathrm{B}$ signalling pathway.

Acetic acid [71] is a common chemical that causes acute colitis. Injection of acetic acid into the colon cavity can cause acute inflammation of colon tissue. TNF-a is closely related to ulcerative colitis. Anise essential oil reduces inflammation by regulating TNF-a levels and inhibiting acetic acid-induced myelin peroxidase activity in tissues [71].

In the LPS-induced in vivo inflammation model, both Angelica essential oil significantly affected the expression of cytokines, inflammatory mediators, and inflammationrelated enzymes in the inflammation rat model. This occurs mainly by regulating the expression of the endothelial adhesion molecules ICAM-1 and VCAM-1, thereby preventing the inflammation induced by LPS.

For establishing skin inflammation models, a variety of chemicals can be used to cause skin inflammation, such as xylene-induced ear oedema, carrageenan-induced paw oedema, DNFB-induced [72] allergic contact dermatitis (ACD), DNCB-induced ear swelling, and AD-like skin lesion models. Essential oils can be used to evaluate the antiinflammatory effects of anti-inflammatory drugs in vivo. Ginger essential oil can protect against xylene-, carrageenanand DNFB-induced cutaneous inflammation. The essential oil from Zanthoxylum coreanum Nakai inhibited DNCBinduced ear swelling and AD-like skin lesions.

3.3. Methodological Quality/Risk of Bias. Figure 3 introduces the methodological features of this review article. The pedigree, frequency of treatment, administration route, and dose of the oils given to experimental animals were clearly described in all the articles. The main objectives and findings of the studies were clearly expressed. However, not all reports reported blind-assessment procedures. Only a few articles reported blind breeding and blind allocation of experimental animals.

Figure 4 and Figure 5 describe the year and country of publication of each article reviewed. Traditional Chinese medicine, as a medicinal material with Chinese herbs, is widely studied not only in China but also in India, South Korea, and Iran. This breadth of study reflects the contribution of traditional Chinese medicine to the development of new drugs around the world. Overall, China has contributed much of value to the world, and traditional Chinese medicine is one of these contributions. Traditional Chinese medicine has been widely used around the world, promoting the development of new drugs based on plant species. As identified by the year of publication, the number of publications has increased gradually in recent years, indicating that the use of traditional Chinese medicine essential oils as anti-inflammatory agents has attracted increasing attention from researchers around the world. 


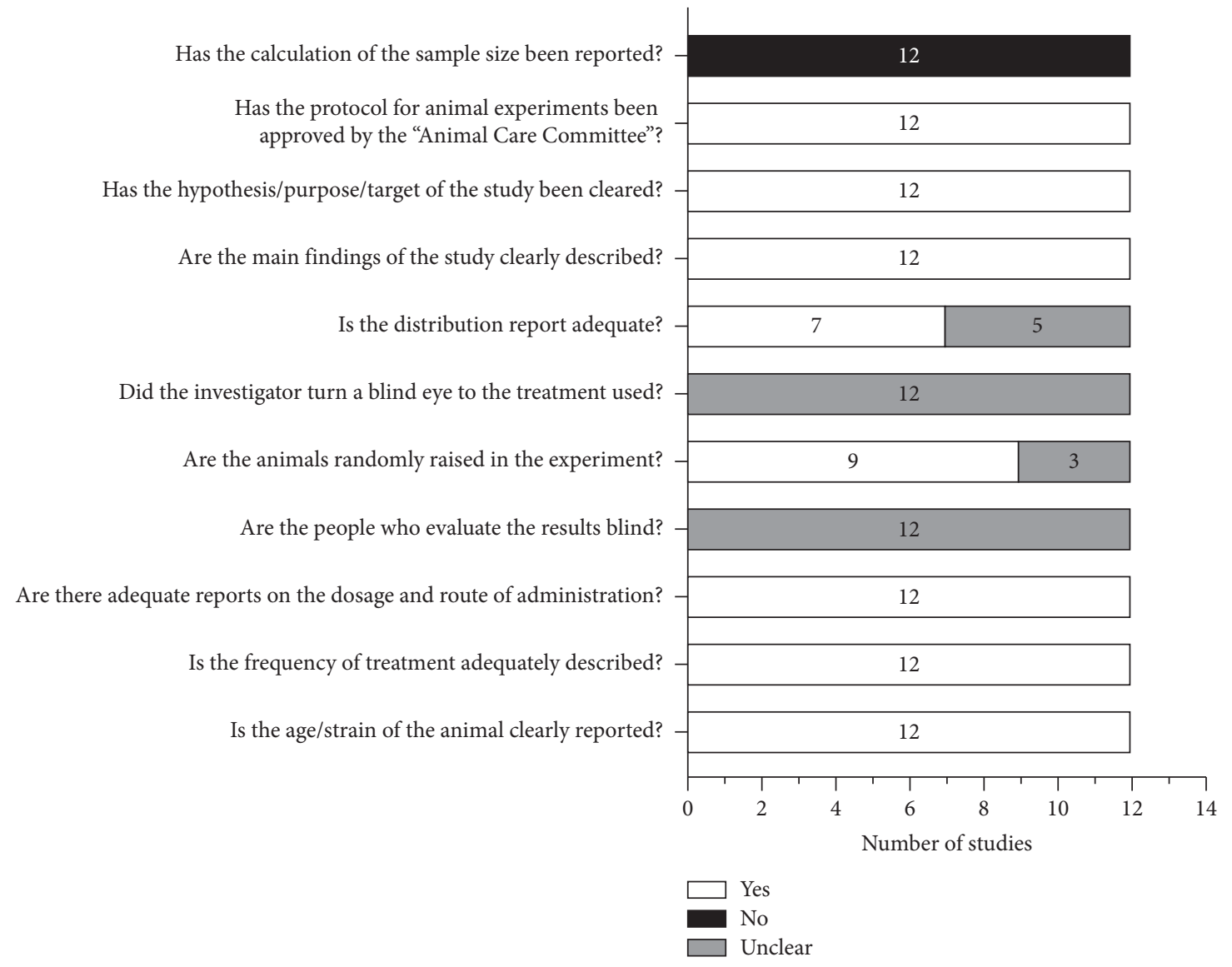

FIGURE 3: Methodological quality of included in vivo studies.

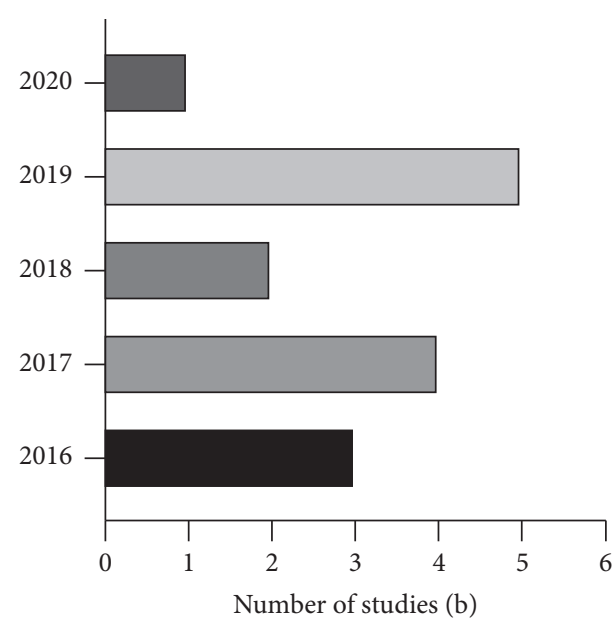

Figure 4: The year of publication of the review article.

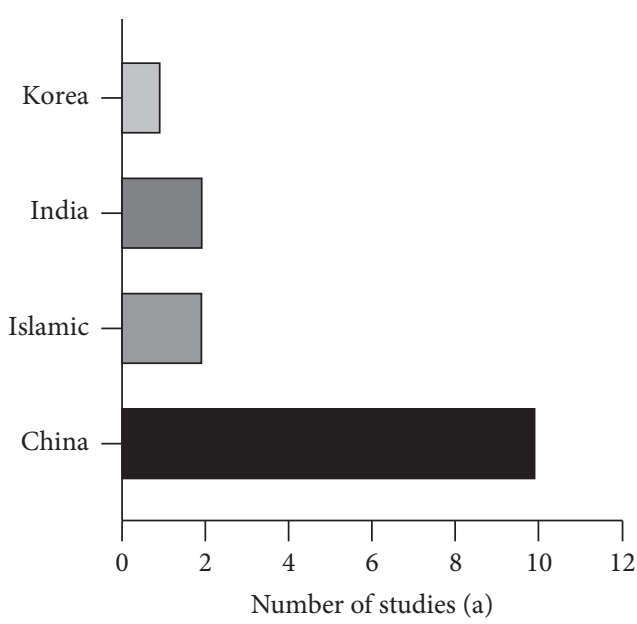

Figure 5: The country of publication of the review article. 


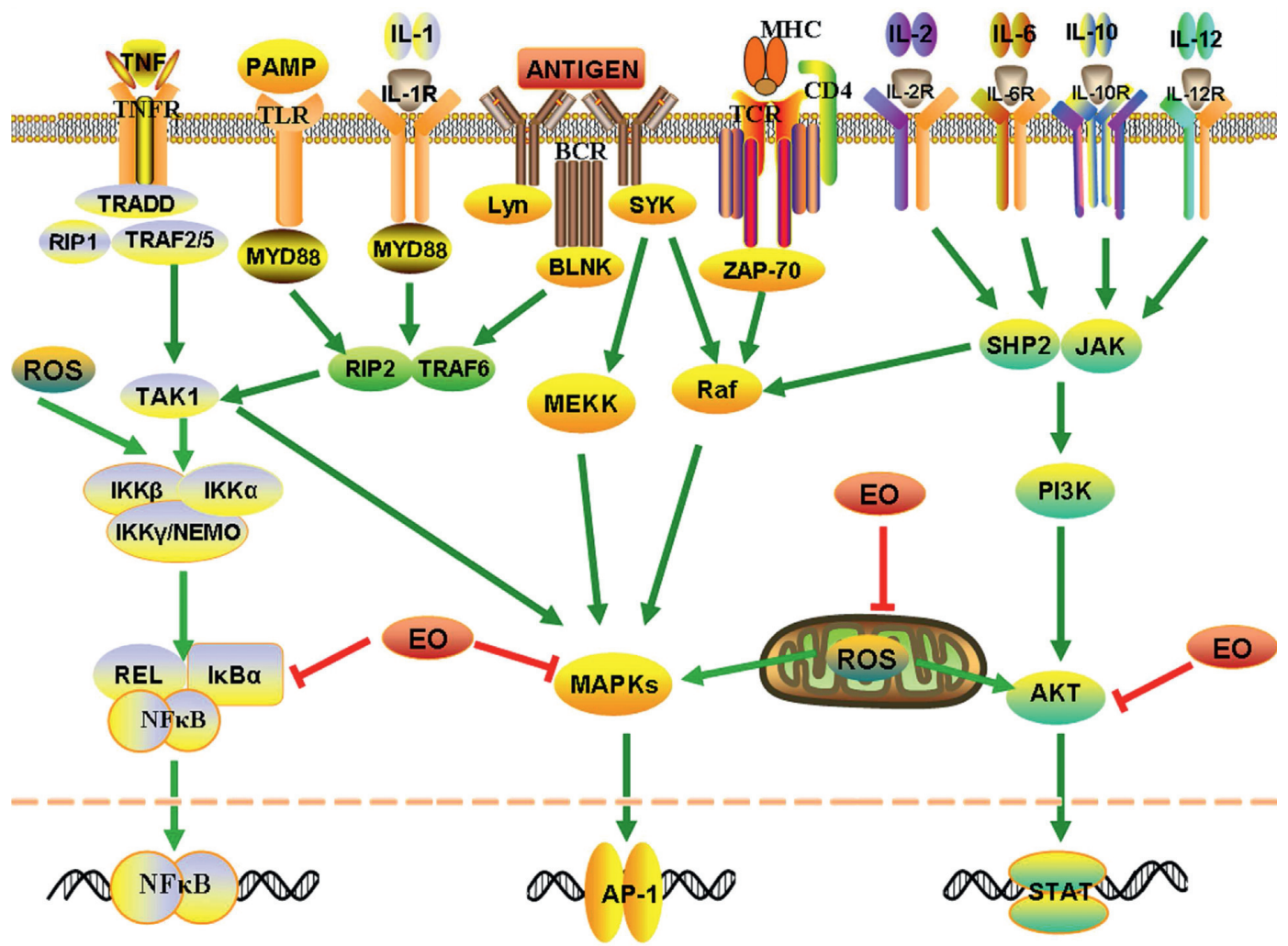

Figure 6: The anti-inflammatory effect of the essential oils of traditional Chinese medicine involves multiple signal pathways.

\section{Conclusion}

This review shows that Chinese herbal oils have great potential as naturally extracted compounds in the treatment of inflammatory diseases. The essential oils of traditional Chinese medicine have shown good antiinflammatory effects in various inflammatory disease models. The essential oils of traditional Chinese medicine can regulate the levels of various cytokines and inhibit multiple signalling pathways that trigger responses to inflammatory diseases, as shown in Figure 6. Most of the articles reviewed in this article were of medium quality, and there are still areas for improvement in some areas. However, overall, the essential oils of traditional Chinese medicine will likely play important roles in the development of new anti-inflammatory drugs in the future.

In 15 articles, we found that essential oils of traditional Chinese medicine have excellent performance in inhibiting signal pathways such as NF- $\kappa \mathrm{B}, \mathrm{MAPK}$, and AKT. Numerous antigens will be recognized by the corresponding T cell receptors, B cell receptors, and Toll-like receptors, cytokines such as TNF and IL will be recognized by TNF receptors and a variety of IL receptors, which will trigger the three inflammatory signal pathways mentioned above. In addition, oxidative stress can also trigger inflammation signalling pathways. These three common inflammatory signal pathways are inhibited, expounding the anti-inflammatory mechanism of traditional Chinese medicine essential oils.

\section{Conflicts of Interest}

The authors declare that they have no conflicts of interest.

\section{Acknowledgments}

This research was supported by the Key Laboratory of Precision Infectious Diseases of Jilin Province (Grant no. 20200601011JC), funded by Development of Plant Antibacterial Peptide Series Health Products (Grant no. 20200404046YY), and funded by Jilin Province Chinese Patent Medicine Secondary Development Technology Innovation Center.

\section{References}

[1] J. Liu, J. Tang, Y. Zuo et al., "Stauntoside B inhibits macrophage activation by inhibiting NF- $\kappa \mathrm{B}$ and ERK MAPK signalling," Pharmacological Research, vol. 111, pp. 303-315, 2016.

[2] K. R. Yu, U. B. Mahajan, B. S. Unger et al., "Animal models of inflammation for screening of anti-inflammatory drugs: implications for the discovery and development of phytopharmaceuticals," International Journal of Molecular Sciences, vol. 20, p. 18, 2019.

[3] A. N. Abdalla, U. Shaheen, Q. M. A. Abdallah et al., "Proapoptotic activity of Achillea membranacea essential oil and its major constituent 1, 8-cineole against A2780 ovarian cancer cells," Molecules, vol. 25, p. 7, 2020.

[4] P. Arulselvan, M. T. Fard, W. S. Tan et al., "Role of antioxidants and natural products in inflammation," Oxidative 
Medicine and Cellular Longevity, vol. 2016, Article ID 5276130, 2016.

[5] D. Simon, "Inflammation and growth," Journal of Pediatric Gastroenterology and Nutrition, vol. 51, no. Suppl 3, pp. S133-S134, 2017.

[6] M. Ebbo, A. Crinier, F. Vély, and E. Vivier, "Innate lymphoid cells: major players in inflammatory diseases," Nature Reviews Immunology, vol. 17, no. 11, pp. 665-678, 2017.

[7] L. Ferrero-Miliani, O. H. Nielsen, P. S. Andersen, and S. E. Girardin, "Chronic inflammation: importance of NOD2 and NALP3 in interleukin-1beta generation," Clin Exp Immunol, vol. 147, no. 2, pp. 227-235, 2007.

[8] S. L. Dahl and J. R. Ward, "Pharmacology, clinical efficacy, and adverse effects of the nonsteroidal anti-inflammatory agent benoxaprofen," Pharmacotherapy: The Journal of Human Pharmacology and Drug Therapy, vol. 2, no. 6, pp. 354-365, 1982.

[9] Y. Li, W. Li, C. Fu, Y. Song, and Q. Fu, "Lonicerae japonicae flos and Lonicerae flos: a systematic review of ethnopharmacology, phytochemistry and pharmacology," Phytochemistry Reviews, vol. 19, no. 1, pp. 1-61, 2019.

[10] H. Lv, Z. Li, Z. Xie et al., "Innovated formulation of TCM pangolin scales to develop a nova therapy of rheumatoid arthritis," Biomedicine \& Pharmacotherapy, vol. 126, p. 109872, 2020.

[11] J. Hu, S. Yuan, X. Wang et al., "Attenuation of pristimerin on TNF- $\alpha$-induced endothelial inflammation," International Immunopharmacology, vol. 82, p. 106326, 2020.

[12] H. Lei, J. Wang, L. Chen, S. Li, and C. Dai, "Comparison of Volatile Oil between the Fruits of Amomum villosum Lour. and Amomum villosum Lour. var. xanthioides T. L. Wu et Senjen Based on GC-MS and Chemometric Techniques," Molecules, vol. 249 pages, 2019.

[13] L. M. L. Campêlo, C. Gonçalves e Sá, A. A. de Almeida et al., "Sedative, anxiolytic and antidepressant activities of Citrus limon (Burn) essential oil in mice," Pharmazie, vol. 66, no. 8, pp. 623-627, 2011.

[14] M. G. Miguel, "Antioxidant and anti-inflammatory activities of essential oils: a short review," Molecules, vol. 15, no. 12, pp. 9252-9287, 2010.

[15] D. Moher, A. Liberati, J. Tetzlaff, and D. G. Altman, "Preferred reporting items for systematic reviews and meta-analyses: the PRISMA statement," PLoS Med, vol. 6, no. 7, Article ID e1000097, 2009.

[16] K.-L. Xu, X.-Q. Gan, Y.-K. Xu et al., "Protective effect of the essential oil of Zanthoxylum myriacanthum var. pubescens against dextran sulfate sodium-induced intestinal inflammation in mice," Phytomedicine, vol. 23, no. 9, pp. 883-890, 2016.

[17] A. Li, K. Agarwal, M. Singh et al., "Essential oil from waste leaves of Curcuma longa L. alleviates skin inflammation," Inflammopharmacology, vol. 26, no. 5, pp. 1245-1255, 2018.

[18] Y.-p. Saxena, T.-y. Shi, Y.-y. Zhang et al., "Essential oil from Fructus Alpinia zerumbet (fruit of Alpinia zerumbet (Pers.) Burtt.et Smith) protected against aortic endothelial cell injury and inflammation in vitro and in vivo," Journal of Ethnopharmacology, vol. 237, pp. 149-158, 2019.

[19] R. H. Lin, J. U. Park, S. J. Jo et al., "Anti-allergic inflammatory effects of the essential oil from fruits of Zanthoxylum coreanum Nakai," Front Pharmacol, vol. 9, p. 1441, 2018.

[20] T. H. Yeh and J. Y. Lin, "Acorus gramineusand and euodia ruticarpa steam distilled essential oils exert anti-inflammatory effects through decreasing Th1/Th2 and pro-/anti- inflammatory cytokine secretion ratios in vitro," Biomolecules, vol. 102 pages, 2020.

[21] X.-L. Huang, X.-J. Li, Q.-F. Qin, Y.-S. Li, W. K. Zhang, and H.-B. Tang, "Anti-inflammatory and antinociceptive effects of active ingredients in the essential oils from Gynura procumbens, a traditional medicine and a new and popular food material," Journal of Ethnopharmacology, vol. 239, p. 111916, 2019.

[22] L.-L. Chen, H.-J. Zhang, J. Chao, and J.-F. Liu, "Essential oil of Artemisia argyi suppresses inflammatory responses by inhibiting JAK/STATs activation," Journal of Ethnopharmacology, vol. 204, pp. 107-117, 2017.

[23] S. Gu, L. Li, H. Huang, B. Wang, and T. Zhang, "Antitumor, antiviral, and anti-inflammatory efficacy of essential oils from atractylodes macrocephala koidz. Produced with different processing methods," Molecules, vol. 2416 pages, 2019.

[24] A. K. Maurya, S. Mohanty, A. Pal, C. S. Chanotiya, and D. U. Bawankule, "The essential oil from Citrus limetta Risso peels alleviates skin inflammation: in-vitro and in-vivo study," Journal of Ethnopharmacology, vol. 212, pp. 86-94, 2018.

[25] C.-Y. Shen, J.-G. Jiang, W. Zhu, and Q. Ou-Yang, "Antiinflammatory effect of essential oil fromCitrus aurantiumL. var.amaraEngl," Journal of Agricultural and Food Chemistry, vol. 65, no. 39, pp. 8586-8594, 2017.

[26] L. Sun, S.-B. Zong, J.-C. Li et al., "The essential oil from the twigs of Cinnamomum cassia Presl alleviates pain and inflammation in mice," Journal of Ethnopharmacology, vol. 194, pp. 904-912, 2016.

[27] N. Lv, H. Lei, X. Li, Q. Wang, M. Liu, and M. Wang, "Protective effects of ginger essential oil (Geo) against chemically-induced cutaneous inflammation," Food Science and Technology, vol. 39, no. suppl 2, pp. 371-377, 2019.

[28] J. Li, Y. Hua, P. Ji et al., "Effects of volatile oils of Angelica sinensis on an acute inflammation rat model," Pharmaceutical Biology, vol. 54, no. 9, pp. 1881-1890, 2016.

[29] N. Yao, H. Jamali, M. Abolhasani, and H. Pazoki Toroudi, "Lavender oil (Lavandula angustifolia) attenuates renal ischemia/reperfusion injury in rats through suppression of inflammation, oxidative stress and apoptosis," Biomedicine \& Pharmacotherapy, vol. 110, pp. 9-19, 2019.

[30] S. M. Rezayat, A.-R. Dehpour, S. M. Motamed et al., "Foeniculum vulgare essential oil ameliorates acetic acid-induced colitis in rats through the inhibition of NF-kB pathway," Inflammopharmacology, vol. 26, no. 3, pp. 851-859, 2018.

[31] X. Zeng, Y. Zhang, J. S. W. Kwong et al., "The methodological quality assessment tools for preclinical and clinical studies, systematic review and meta-analysis, and clinical practice guideline: a systematic review," Journal of Evidence-Based Medicine, vol. 8, no. 1, pp. 2-10, 2015.

[32] J. Zhang, A. Koeberle, F. Dehm et al., "Arzanol, a prenylated heterodimeric phloroglucinyl pyrone, inhibits eicosanoid biosynthesis and exhibits anti-inflammatory efficacy in vivo," Biochemical Pharmacology, vol. 81, no. 2, pp. 259-268, 2011.

[33] N. E. Burma, H. Leduc-Pessah, C. Y. Fan, and T. Trang, "Animal models of chronic pain: advances and challenges for clinical translation," Journal of Neuroscience Research, vol. 95, no. 6, pp. 1242-1256, 2017.

[34] A. T. Rufino, M. Ribeiro, F. Judas et al., "Anti-inflammatory and chondroprotective activity of $(+)-\alpha$-Pinene: structural and enantiomeric selectivity," Journal of Natural Products, vol. 77, no. 2, pp. 264-269, 2014.

[35] C.-M. Salgueiro and J. Y. Lin, "Anti-inflammatory effects of 27 selected terpenoid compounds tested through modulating Th1/ 
Th2 cytokine secretion profiles using murine primary splenocytes," Food Chemistry, vol. 141, no. 2, pp. 1104-1113, 2013.

[36] X.-J. Li, Y.-J. Yang, Y.-S. Li, W. K. Zhang, and H.-B. Tang, " $\alpha$-Pinene, linalool, and 1-octanol contribute to the topical anti-inflammatory and analgesic activities of frankincense by inhibiting COX-2," Journal of Ethnopharmacology, vol. 179, pp. 22-26, 2016.

[37] M. T. Chen, Y. J. Yang, Y. S. Li et al., "Shengfu oil enhances the healing of full-thickness scalded skin accompanying the differential regulation of $\beta$-catenin, Dlk1, and COX-2," Frontier Pharmacology, vol. 8, p. 801, 2017.

[38] X. Bao, H. Yuan, C. Wang, J. Liu, and M. Lan, "Antitumor and immunomodulatory activities of a polysaccharide from Artemisia argyi," Carbohydrate Polymers, vol. 98, no. 1, pp. 1236-1243, 2013.

[39] X. Kou, S. Qi, W. Dai, L. Luo, and Z. Yin, “Arctigenin inhibits lipopolysaccharide-induced iNOS expression in RAW264.7 cells through suppressing JAK-STAT signal pathway," International Immunopharmacology, vol. 11, no. 8, pp. 10951102, 2011.

[40] M. M. Ehrnhöfer-Ressler, K. Fricke, M. Pignitter et al., "Identification of 1,8-cineole, borneol, camphor, and thujone as anti-inflammatory compounds in aSalvia officinalisL. Infusion using human gingival fibroblasts," Journal of Agricultural and Food Chemistry, vol. 61, no. 14, pp. 3451-3459, 2013.

[41] D. C. Walker and S. R. Carding, "Inflammatory bowel disease: cause and immunobiology," The Lancet, vol. 369, no. 9573, pp. 1627-1640, 2007.

[42] G.-Y. Kim, K.-H. Kim, S.-H. Lee et al., "Curcumin inhibits immunostimulatory function of dendritic cells: MAPKs and translocation of NF- $\kappa \mathrm{B}$ as potential targets," The Journal of Immunology, vol. 174, no. 12, pp. 8116-8124, 2005.

[43] C.-Y. Yoon, L. Yang, J.-G. Jiang, C.-Y. Zheng, and W. Zhu, "Immune enhancement effects and extraction optimization of polysaccharides from Citrus aurantium L. var. amara Engl," Food \& Function, vol. 8, no. 2, pp. 796-807, 2017.

[44] X. Yao, D. Wu, N. Dong, et al.,C. Moracin, "A phenolic compound isolated from artocarpus heterophyllus, suppresses lipopolysaccharide-activated inflammatory responses in murine Raw264.7 macrophages," International Journal of Molecular Sciences, vol. 17, no. 8, 2016.

[45] I.-N. Hsieh, A. Chang, C.-M. Teng, C.-C. Chen, and C.-R. Yang, "Aciculatin inhibits lipopolysaccharide-mediated inducible nitric oxide synthase and cyclooxygenase- 2 expression via suppressing NF- $\kappa \mathrm{B}$ and JNK/p38 MAPK activation pathways," Journal of Biomedical Science, vol. 18, no. 1, p. 28, 2011.

[46] G. H. da Cunha, M. O. de Moraes, F. V. Fechine et al., "Vasorelaxant and antihypertensive effects of methanolic fraction of the essential oil of Alpinia zerumbet," Vascular Pharmacology, vol. 58, no. 5-6, pp. 337-345, 2013.

[47] T. Zhu, D. X. Wang, W. Zhang et al., "Andrographolide protects against LPS-induced acute lung injury by inactivation of NF- $\kappa \mathrm{B}$," PLoS One, vol. 8, no. 2, Article ID e56407, 2013.

[48] A. Gisterå and G. K. Hansson, "The immunology of atherosclerosis," Nature Reviews Nephrology, vol. 13, no. 6, pp. 368-380, 2017.

[49] A. L. Eikendal, A. M. Evelein, C. S. Uiterwaal et al., "Relation between circulating inflammatory chemokines and vascular characteristics in healthy, young children," Journal of the American Heart Association, vol. 4, p. 12, 2015.

[50] C. Poussin, I. Gallitz, W. K. Schlage et al., "Mechanism of an indirect effect of aqueous cigarette smoke extract on the adhesion of monocytic cells to endothelial cells in an in vitro assay revealed by transcriptomics analysis," Toxicology in Vitro, vol. 28, no. 5, pp. 896-908, 2014.

[51] R. Steffen, Y. Xu, H. Fu, J. Wang, L. Jin, and S. Li, "Urocortin induced expression of COX-2 and ICAM-1 via corticotrophin-releasing factor type 2 receptor in rat aortic endothelial cells," British Journal of Pharmacology, vol. 158, no. 3, pp. 819-829, 2009.

[52] F. Tang, F. Chen, X. Ling et al., "Inhibitory effect of methyleugenol on IgE-mediated allergic inflammation in RBL-2H3 cells," Mediators Inflammation, p. 463530, 2015.

[53] H. J. Do, T. W. Oh, J. H. Yang, K. I. Park, and J. Y. Ma, "Davallia mariesii moore improves FceRI-mediated allergic responses in the rat basophilic leukemia mast cell line RBL$2 \mathrm{H} 3$ and passive cutaneous anaphylaxis in mice," Mediators Inflammation, vol. 2017, Article ID 8701650, 9 pages, 2017.

[54] E.-H. Song, K.-S. Chung, Y.-M. Kang, J.-H. Lee, M. Lee, and H.-J. An, "Eupatilin suppresses the allergic inflammatory response in vitro and in vivo," Phytomedicine, vol. 42, pp. 1-8, 2018.

[55] T. S. Kupper and R. C. Fuhlbrigge, "Immune surveillance in the skin: mechanisms and clinical consequences," Nature Reviews Immunology, vol. 4, no. 3, pp. 211-222, 2004.

[56] I. M. Carrara, G. P. Melo, S. S. Bernardes et al., "Looking beyond the skin: cutaneous and systemic oxidative stress in UVB-induced squamous cell carcinoma in hairless mice," Journal of Photochemistry and Photobiology B: Biology, vol. 195, pp. 17-26, 2019.

[57] S. Neto, A. Ray, A. Banerjee et al., "Chemical composition and antioxidant activity of essential oil from leaves and rhizomes of Curcuma angustifolia Roxb," Natural Product Research, vol. 31, no. 18, pp. 2188-2191, 2017.

[58] W.-J. Sahoo, N. H. Lee, and C.-G. Hyun, "Limonene suppresses lipopolysaccharide-induced production of nitric oxide, prostaglandin E2, and proinflammatory cytokines in RAW 264.7 macrophages," Journal of Oleo Science, vol. 59, no. 8, pp. 415-421, 2010.

[59] L. S. Hoang, M. H. Tran, J.-S. Lee, Q. M. T. Ngo, M. H. Woo, and B. S. Min, "Inflammatory inhibitory activity of sesquiterpenoids from atractylodes macrocephala rhizomes," Chemical and Pharmaceutical Bulletin, vol. 64, no. 5, pp. 507-511, 2016.

[60] J. Zhang, G. Cao, Y. Xia, C. Wen, and Y. Fan, "Fast analysis of principal volatile compounds in crude and processed Atractylodes macrocephala by an automated static headspace gas chromatography-mass spectrometry," Pharmacognosy Magazine, vol. 10, no. 39, pp. 249-253, 2014.

[61] H.-Y. Kim, S.-Y. Nam, S.-Y. Hwang, H.-M. Kim, and H.-J. Jeong, "Atractylone, an active constituent of KMP6, attenuates allergic inflammation on allergic rhinitis in vitro and in vivo models," Molecular Immunology, vol. 78, pp. 121-132, 2016.

[62] Z. Guo, Z. Liu, H. Yue, and J. Wang, "Beta-elemene increases chemosensitivity to 5-fluorouracil through down-regulating microRNA-191 expression in colorectal carcinoma cells," Journal of Cellular Biochemistry, vol. 119, no. 8, pp. 70327039, 2018.

[63] J. Chen, T. Wang, S. Xu et al., "Novel hybrids of natural $\beta$ -elemene bearing isopropanolamine moieties: synthesis, enhanced anticancer profile, and improved aqueous solubility," Fitoterapia, vol. 120, pp. 117-125, 2017.

[64] Y. Lin, Y. Zhang, W. Gu, L. He, and B. Sun, “Th1/Th2 cell's function in immune system," Advances in Experimental Medicine and Biology, vol. 841, pp. 45-65, 2014. 
[65] N. Gagliani and S. Huber, "Basic aspects of T helper cell differentiation," Methods in Molecular Biology, vol. 1514, pp. 19-30, 2017.

[66] H. Inoue, T. Mori, S. Shibata, and Y. Koshihara, "Modulation by glycyrrhetinic acid derivatives of TPA-induced mouse ear oedema," British Journal of Pharmacology, vol. 96, no. 1, pp. 204-210, 1989.

[67] A. K. Verma, D. L. Wheeler, M. H. Aziz, and H. Manoharan, "Protein kinase $\mathrm{C} \varepsilon$ and development of squamous cell carcinoma, the nonmelanoma human skin cancer," Molecular Carcinogenesis, vol. 45, no. 6, pp. 381-388, 2006.

[68] J. A. Udobang, P. A. Nwafor, and J. E. Okokon, "Analgesic and antimalarial activities of crude leaf extract and fractions of Acalypha wilkensiana," Journal of Ethnopharmacology, vol. 127, no. 2, pp. 373-378, 2009.

[69] H. R. Pazoki-Toroudi, M. Ajami, and R. Habibey, "Premedication and renal pre-conditioning: a role for alprazolam, atropine, morphine and promethazine," Fundamental \& Clinical Pharmacology's, vol. 24, no. 2, pp. 189-198, 2010.

[70] S. Wirtz, C. Neufert, B. Weigmann, and M. F. Neurath, "Chemically induced mouse models of intestinal inflammation," Nature Protocols, vol. 2, no. 3, pp. 541-546, 2007.

[71] N. Goyal, A. Rana, A. Ahlawat, K. R. V. Bijjem, and P. Kumar, "Animal models of inflammatory bowel disease: a review," Inflammopharmacology, vol. 22, no. 4, pp. 219-233, 2014.

[72] Y.-L. Shi, J. Gu, J.-Y. Park et al., "Histone deacetylases inhibitor Trichostatin A ameliorates DNFB-induced allergic contact dermatitis and reduces epidermal Langerhans cells in mice," Journal of Dermatological Science, vol. 68, no. 2, pp. 99-107, 2012. 